\title{
Lymphatic Capillary
}

National Cancer Institute

\section{Source}

National Cancer Institute. Lymphatic Capillary. NCI Thesaurus. Code C33037.

Tiny vessels with porous walls that join to form the lymphatic vessels. 